\title{
Robust detection of CID double stars in SDSS ${ }^{\star}$
}

\author{
D. Pourbaix ${ }^{1, \star \star}$, G. R. Knapp ${ }^{2}$, J. E. Gunn ${ }^{2}$, R. H. Lupton ${ }^{2}$, Ž. Ivezić ${ }^{3}$, C. Siopis $^{1}$, M. Rigaux ${ }^{4}$, and A. Rubbens ${ }^{5}$ \\ ${ }^{1}$ Institut d'Astronomie et d'Astrophysique, Université Libre de Bruxelles (ULB), 1050 Brussels, Belgium \\ e-mail: pourbaix@astro.ulb.ac.be \\ 2 Department of Astrophysical Sciences, Princeton University, Princeton, NJ 08544-1001, USA \\ 3 Department of Astronomy, University of Washington, Seattle, WA 98195-1580, USA \\ 4 Département de Physique, Université Libre de Bruxelles (ULB), 1050 Brussels, Belgium \\ 5 Collège Saint-Hubert, 1170 Brussels, Belgium
}

Received 11 April 2016/ Accepted 11 May 2016

\begin{abstract}
Aims. The Sloan Digital Sky Survey (SDSS) offers a unique possibility of not only detecting colour induced displacement (CID) double stars but also confirming these detections.

Methods. Successive cuts are applied to the SDSS data release (DR) 12 database to reduce the size of the sample under consideration. The resulting dataset is then screened with a criterion based on the distance and orientation of the photocentres in different photometric bands.

Results. About 3200 distinct objects are classified as CID double stars, 40 of which are confirmed with at least a second detection. A consistency check further validates these detections.
\end{abstract}

Key words. binaries: general - astrometry - methods: data analysis

\section{Introduction}

The Sloan Digital Sky Survey (SDSS; York et al. 2000; Alam et al. 2015, and references therein) has revolutionised stellar astronomy since the late 90 s by providing homogeneous and deep $(r<22.5)$ photometry in five passbands $(u, g$, $r, i$, and $z$; Fukugita et al. 1996; Gunn et al. 1998; Hogg et al. 2001; Smith et al. 2002; Doi et al. 2010) accurate to $1-2 \%$ (Padmanabhan et al. 2008). The sky coverage, $14555 \mathrm{deg}^{2}$ in the Northern Galactic Cap, results in photometric measurements for over 260 million stars and 208 million galaxies. Astrometric positions are accurate to better than 0.1 arcsec per coordinate (rms) for point sources with $r<20.5^{\mathrm{m}}$ (Pier et al. 2003), and the morphological information from the images allows robust stargalaxy separation to $r \sim 21.5^{\mathrm{m}}$ (Lupton et al. 2003). The successive SDSS data releases (e.g., Abazajian et al. 2003, 2004, 2005; Adelman-McCarthy et al. 2007) have provided the position of an increasing number of stars in the five photometric bands. Up until SDSS DR7 (Abazajian et al. 2009), the positions at only one epoch were available for any given object. Since SDSS DR8 (Aihara et al. 2011), repeated observations have been reported.

SDSS has been used extensively for extragalactic investigations, however other groups have taken advantage of it for stellar astrophysical purposes, especially binaries (Silvestri et al. 2007; Clark et al. 2012). Thanks to the multiple photometric bands, colour-colour outlier detection was the first method adopted to filter the double stars out (Raymond et al. 2003; Smolčić et al. 2004; Augusteijn et al. 2008; Liu et al. 2012). The photometric and spectroscopic capabilities of SDSS were also combined

\footnotetext{
^ Full Tables 1 and 2 are only available at the CDS via anonymous ftp to cdsarc.u-strasbg. fr $(130.79 .128 .5)$ or via

http://cdsarc.u-strasbg.fr/viz-bin/qcat?]/A+A/591/A96

$\star \star$ Senior Research Associate, F.R.S.-FNRS, Belgium.
}

to detect photometrically well-behaved objects (Szkody et al. 2002, 2003a,b, 2004, 2005), including post-common envelope binaries (Schreiber et al. 2010; Nebot Gómez-Morán et al. 2011; Rebassa-Mansergas et al. 2012a). Spectroscopic binaries have also been detected thanks to the variability of their radial velocity (Pourbaix et al. 2005; Morganson et al. 2015).

SDSS pairs composed of a white dwarf and a main sequence (typically M) star have been quite intensively investigated (see Rebassa-Mansergas et al. 2012b, and references therein) over the past $10 \mathrm{yr}$. These systems offer the combination of two stars at very distinct stages of their evolution but with a similar brightness, and distinctive colours. Their value for our understanding of stellar evolution is therefore what also makes them rather easy to detect through unusual colours.

The detection of unresolved double stars through the wavelength dependence of the position of the photocentre (colour induced displacement double stars, CID) was suggested by Christy et al. (1983) and Sorokin \& Tokovinin (1985) and successfully applied to the SDSS DR2 and DR5 observations (Pourbaix et al. 2004; Pourbaix 2008). The same technique has lately been applied to the USNO-B1.0 dataset (Jayson 2016). However, in all these investigations, the positions were measured at one epoch only, thus preventing any confirmation of what could simply be a false detection. Although the detection of CID double stars requires $2+$ photometric filters, it only relies upon the position measured through these filters, not the colour itself. This method can therefore be applied to probe the whole stellar locus for binaries, with the exception of twins. The more distinct the colour of the components, the farther apart their photocentres, so white dwarf $+M$ dwarf are, again, among the privileged systems. However, assuming the astrometry through the two filters can be tied up, there is no constraint on the type of the 
Table 1. Identified CID with their thingId, position, and SDSS dereddened magnitude for the five photometric bands.

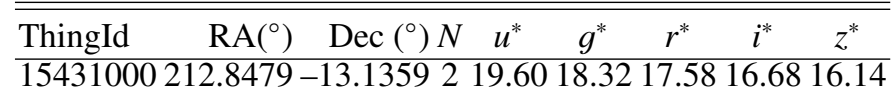

Notes. $N$ is the number of observations flagged as CID. The full table is available at the CDS.

components since the detection capability is simply limited by the astrometric precision.

Taking advantage of the availability of repeated observations since DR8, we here present the multiple detection of CID double stars based on public SDSS data only. The initial selection of the sample is described in Sect. 2. The CID criteria to be fulfilled are described in Sect. 3. The nature of the components is considered in Sect. 4. Finally, the time variability of the CID feature is analysed in Sect. 5.

\section{Observational data}

All the data to be analysed come from one single table, PhotoObjAll, which contains more than one billion rows. The number of rows has remained unchanged for the past five data releases but some bug fixes might have occurred so, from now on, DR12 data (Alam et al. 2015) are going to be assumed. A significant improvement introduced in DR9 from the viewpoint of this investigation is related to the differential chromatic correction (DCR). Pier et al. (2003) describe how DCR is calibrated and corrected but the DR9 astrometry page ${ }^{1}$ mentions that DCR has only been fully accounted for from DR9 onwards. The results of Pourbaix (2008) were therefore still affected by some colour terms that were unaccounted for.

Successive selections are required to clean up the sample initially composed of PhotoObjAll. Only the observations of objects belonging to the Star view are considered. The same filtering as introduced by Pourbaix et al. (2004) was applied: an observation was removed if any of the flags saturated, bright, edge, or nodeblend was set or if the precision on $u, g, r, i$, or $z$ was larger than $0.1,0.05,0.1,0.05$, and 0.05 respectively.

There are 25797735 stars that fulfil these criteria. For them, the standard deviation of the offsets with respect to the $r$ position in $z$ for both $\alpha \cos \delta$ and $\delta$ are 21 mas. For the $u$-band, the standard deviations in $\alpha \cos \delta$ and $\delta$ are 42 mas and 44 mas respectively. These four values are based on the central $99 \%$ of the offsets. Despite this $1 \%$ clipping, the scatter of the offsets in $u$ is $30 \%$ larger than derived by Pier et al. (2003). Both the right ascensions (together) and declinations (together) are correlated at the level 0.16 whereas the correlation between any right ascension and any declination is always 1 order of magnitude smaller.

\section{CID filtering}

The idea behind CID double stars is that the wavelengthdependent photocentres should be aligned along the two stars rather than being randomly distributed around some median position (Pourbaix et al. 2004). Even though the positions in two photometric bands are enough to notice a displacement (Jayson 2016), three positions are necessary to assess the alignment. Among the five photometric bands from SDSS, $u$ and $z$ are the furthest apart in terms of wavelength, whereas $r$ is more central.

\footnotetext{
1 https://wwW.sdss3.org/dr9/algorithms/astrometry.php
}
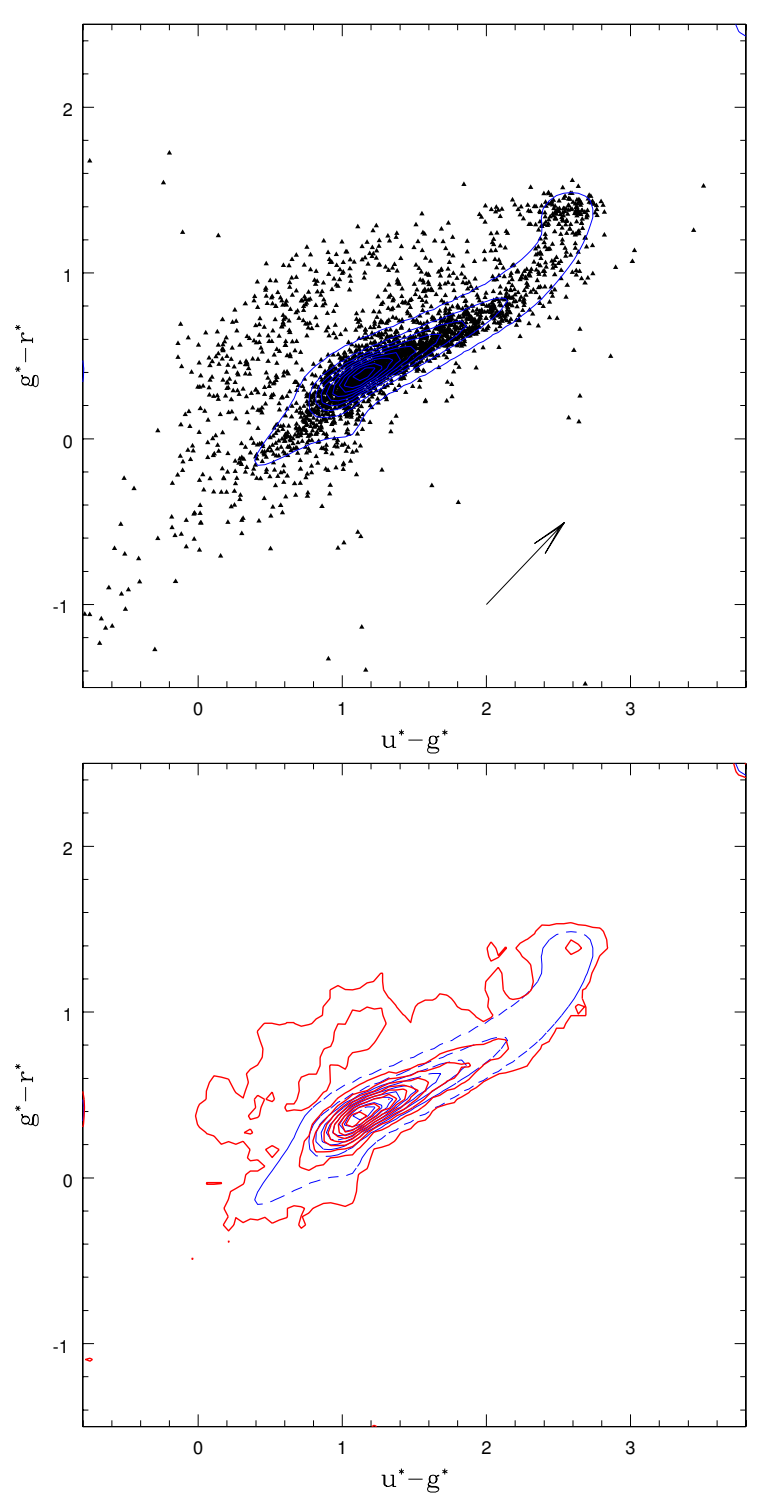

Fig. 1. Top panel: position of the CID candidates in the dereddened colour-colour diagram together with the stellar locus based on 26 million Star colours (i.e. 10\% of the stellar content of SDSS). The arrow denotes the extinction (Schlafly \& Finkbeiner 2011). Bottom panel: contour lines for the CID candidates (thick) and $10 \%$ of the stellar content of SDSS (thin dashed).

The results of the previous section used in a simulation reveal that about one single star out of 600000 could accidentally have its $u$ and $z$ positions more than $0.4^{\prime \prime}$ apart and aligned to more than $177.5^{\circ}$ (in fact, cosine lower than -0.999). In a sample of 10 million observations, we thus anticipate 16 of these false detections. However, the probability of the same single star being observed twice with the same features in its positions is about $3 \times 10^{-12}$.

There are 3273 stellar observations that match the criteria on separation $\left(>=0.4^{\prime \prime}\right)$, orientation $\left(>=177.5^{\circ}\right)$ and photometric quality. They correspond to 3212 distinct objects (Table 1) whose accepted CID observations are listed in Table 2. The location of these points in a dereddened colour-colour diagram is plotted in Fig. 1. For the sake of comparison, the stellar locus based on $10 \%$ of the Star entries, with the same photometric properties (ranges and precisions) as the CID candidates is also plotted. Even though the photometric distribution of the CID 
Table 2. Accepted CID observations for the candidates listed in Table 1.

\begin{tabular}{lllclcc}
\hline \hline ThingId & objectid & MJD & $\begin{array}{c}\Delta \alpha_{u}^{*} \\
\left({ }^{\prime \prime}\right)\end{array}$ & $\begin{array}{c}\Delta \delta_{u} \\
\left({ }^{\prime \prime}\right)\end{array}$ & $\begin{array}{c}\Delta \alpha_{z}^{*} \\
\left({ }^{\prime \prime}\right)\end{array}$ & $\begin{array}{c}\Delta \delta_{z} \\
\left({ }^{\prime \prime}\right)\end{array}$ \\
\hline 523314 & 1237668627303039488 & 53527.23 & -0.0081 & +0.0111 & +0.2453 & -0.3173 \\
\hline
\end{tabular}

Notes. The table lists the thingId, objectId, date, and offsets in right ascension $\left(\alpha^{*}=\alpha \cos \delta\right)$, and declination in the $u$ and $z$-bands with respect to the position in the $r$-band. The full table is available at the CDS.

Table 3. Confirmed CID candidates.

\begin{tabular}{|c|c|c|c|c|}
\hline ThingId & $\mathrm{RA}\left({ }^{\circ}\right)$ & $\operatorname{Dec}\left({ }^{\circ}\right)$ & $N$ & Spec \\
\hline 15431000 & 212.847899 & -13.135926 & 2 & \\
\hline 22550603 & 117.733729 & -10.479184 & 2 & \\
\hline 45706433 & 324.637702 & -5.054417 & 2 & \\
\hline 47860353 & 324.727576 & -4.391773 & 2 & \\
\hline 68189740 & 5.546124 & -1.128588 & 3 & M4 \\
\hline 69946683 & 320.716211 & -1.097230 & 2 & \\
\hline 74734108 & 94.881818 & -0.996007 & 2 & \\
\hline 83883365 & 323.069439 & -0.426253 & 3 & \\
\hline 84696684 & 57.788778 & -0.480402 & 2 & \\
\hline 85303451 & 351.483366 & -0.499383 & 2 & \\
\hline 93807459 & 70.608754 & -0.206033 & 3 & \\
\hline 94010862 & 47.522422 & -0.050319 & 2 & \\
\hline 94025347 & 94.805658 & -0.158900 & 2 & \\
\hline 96981424 & 17.849609 & 0.159764 & 5 & M2* \\
\hline 103499550 & 74.703028 & 0.215468 & 2 & \\
\hline 106358786 & 15.923339 & 0.525698 & 3 & $\mathrm{M} 2 *$ \\
\hline 107874362 & 346.798161 & 0.477047 & 2 & \\
\hline 115775052 & 13.577270 & 0.962821 & 4 & M4* \\
\hline 116724303 & 81.454729 & 1.005443 & 4 & \\
\hline 116891499 & 358.869452 & 1.006782 & 2 & M1 \\
\hline 116995966 & 348.090035 & 1.024174 & 5 & M3* \\
\hline 119480169 & 324.594285 & 1.062909 & 2 & \\
\hline 121325158 & 15.037479 & 1.142725 & 4 & M1* \\
\hline 121351015 & 21.482147 & 1.075074 & 4 & \\
\hline 121561453 & 55.658241 & 1.149527 & 3 & M3* \\
\hline 122233016 & 9.736654 & 1.114130 & 3 & M4 \\
\hline 132088747 & 261.203461 & 2.253603 & 2 & \\
\hline 184456091 & 27.072581 & 8.015645 & 2 & \\
\hline 186374149 & 203.360705 & 8.254411 & 2 & \\
\hline 200646968 & 126.409171 & 9.857400 & 2 & \\
\hline 220442434 & 125.558020 & 12.243735 & 2 & M3* \\
\hline 259525347 & 251.906928 & 16.610724 & 2 & \\
\hline 274459969 & 236.094783 & 18.549545 & 2 & \\
\hline 337204518 & 120.918335 & 25.924343 & 2 & \\
\hline 395512014 & 341.514497 & 33.791220 & 3 & \\
\hline 405351640 & 121.363021 & 35.285873 & 2 & \\
\hline 429504180 & 253.606452 & 39.296835 & 2 & \\
\hline 451341367 & 122.130724 & 43.010222 & 2 & M4.5III* \\
\hline 486944679 & 247.731754 & 50.049775 & 2 & \\
\hline 490371322 & 133.634605 & 50.857572 & 2 & M4.5III* \\
\hline
\end{tabular}

Notes. ThingId denotes the unique SDSS identifier, RA and Dec are the right ascension and declination (2000.0). $N$ is the number of observations flagged as CID. Spec is the optical spectral classification listed by the DR12 Science Archive Server, * indicates the presence of some prominent Balmer lines.

candidates essentially overlap with the locus of the regular stars, it also leaks on the upper left region of the latter (Fig. 1). We come back to this point in Sect. 4 .

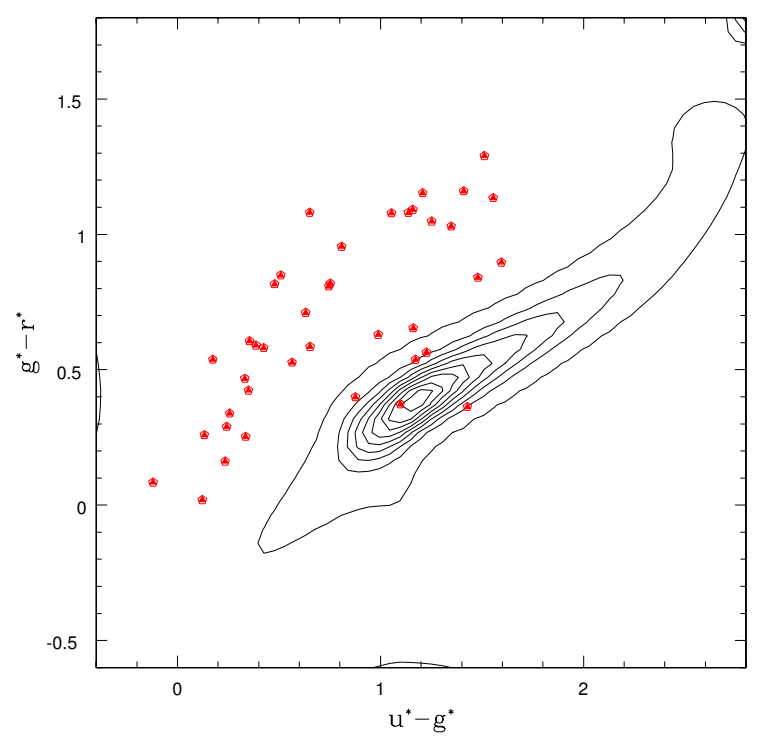

Fig. 2. Position of the confirmed CID candidates in the dereddened colour-colour diagram together with the stellar locus.

In terms of distribution over the sky, there is a small excess of CID detections close to the equator with respect to the parent population. That is however consistent with a criterion partly based on $\Delta \alpha \cos \delta$, where $\Delta \alpha$ stands for the difference of offsets in right ascension in the $u$ and $z$-bands. For an object close to the equator, that quantity and, therefore, the separation between the $u$ and $z$ photocentres are more likely to exceed any adopted threshold.

So far, the adopted methodology is the same as in Pourbaix et al. (2004) and the change in the number of candidates is only caused by the substantial increase of the number of stars observed. Since DR8, some stars have been observed on several occasions, making possible the search for CID candidates among them. It is worth noting that a detection does not necessarily mean a photometric observation (valid or not). The number of observations stored in PhotoObjAll thus often turns out to be lower than publicised by the field nDetect. For instance, if we consider the stars detected 5+ times, they account for nearly 76 million detections but PhotoObjAll only contains 66 million observations for them. By further imposing that a star was detected more than once, we are left with 40 objects with $2+$ CID-like observations (i.e. with a much lower risk of being false detections). The position of these confirmed CID candidates in the colour-colour diagram together with the stellar locus is plotted in Fig. 2. Their location in the sky is listed in Table 3.

\section{Tentative nature of the components}

As already stated in Sect. 3, the $\left(u^{*}-g^{*}, g^{*}-r^{*}\right)$ locus of the CID overlaps with the single star that has a distinctive leakage towards the upper left corner of the latter. The contour line shows 


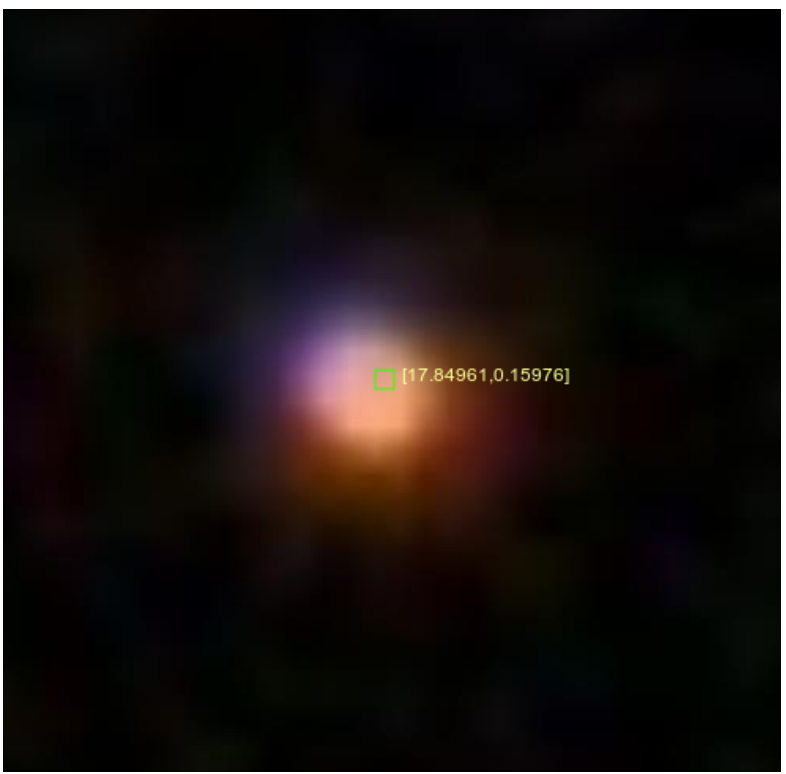

Fig. 3. Image of 96981424 (SDSS J011123.90+000935.1) exhibits a colour gradient whose orientation seems to be orthogonal to the CID.

that the flooding is not continuous but instead represents a bridge between the $\mathrm{M}$ and white dwarfs (Smolčić et al. 2004). The three CID depicted in Fig. 6 belong to this category.

A second leakage of the single star locus in its lower left end results from an overcorrection of the galactic extinction (Schlegel et al. 1998) for objects close to the galactic plane. Regardless of how unrealistic some of these dereddened colours are, they cannot be responsible for the CID status. Indeed, the criteria for being considered as a CID are purely astrometric. Even though some spurious astrometric results can sometime come from a wrong chromatic correction (Pourbaix et al. 2003), any chromatic correction is based on the observed colours, not the dereddened ones. So, even if some dereddened colours might be wrong, the positions should nevertheless always be accurate.

With respect to the single star stellar locus, the CID candidates exhibit an excess of M-dwarf-like objects centred in (2.6, 1.4) on the $\left(u^{*}-g^{*}, g^{*}-r^{*}\right)$ density map (Fig. 1). According to West et al. (2008), their riz colours correspond to spectral types ranging from $\mathrm{M} 0$ to $\mathrm{M} 4$.

Among the 40 confirmed CID, only 12 got their spectral type directly determined through spectroscopy (Stoughton et al. 2002; Bolton et al. 2012). All are M-type: ten dwarfs and two giants. In seven cases, some prominent Balmer lines are detected in absorption, which are hints of the presence of a white dwarf in the same spectroscopic field of view. Over the years and the data releases, several groups have published lists of white dwarfs, M-dwarfs, and binaries with a white dwarf (Heller et al. 2009; Rebassa-Mansergas et al. 2010; West et al. 2011; Kleinman et al. 2013). In total, ten of our candidates belong to at least one of these lists. The remaining 30 objects (including the two with an $\mathrm{M}$ giant component) are still completely absent from any published investigation (according to Simbad). In particular, none of the five objects that belong to the stellar locus in Fig. 2 has had its spectral type determined in the framework of SDSS, nor by any other investigation.

For 25 objects for which an image is available, that image, though point-like, clearly shows two regions of distinct colours, thus leading to a distinct position for the photocentre in at least two photometric bands (e.g. thingId \#96981424, Fig. 3). All the

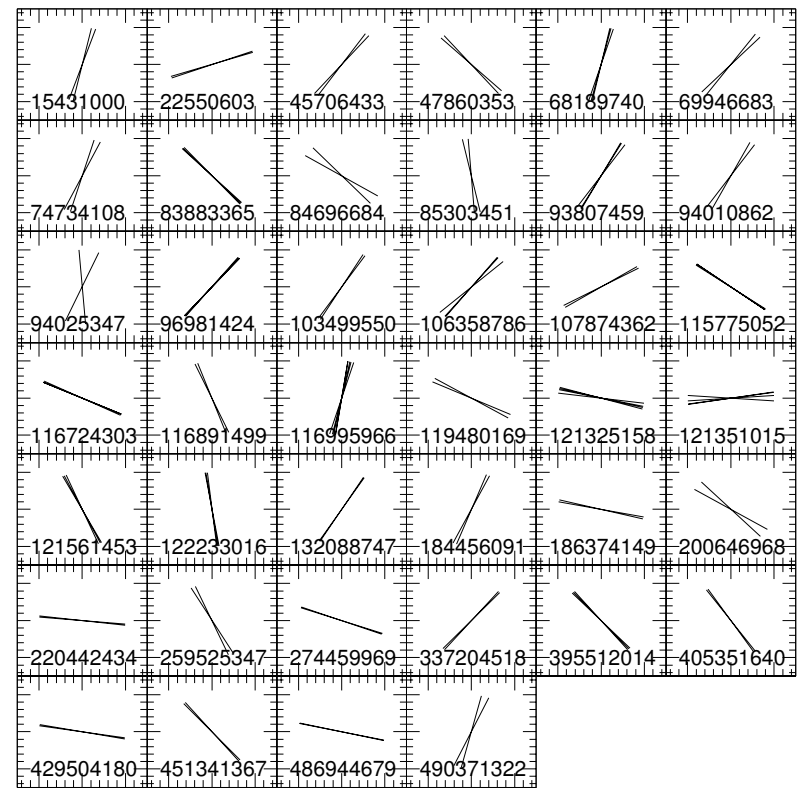

Fig. 4. Orientation of the lines joining the $u$ and $z$ positions of the 40 double stars with $2+$ CID-like observations. The numbers are the SDSS thingid field.

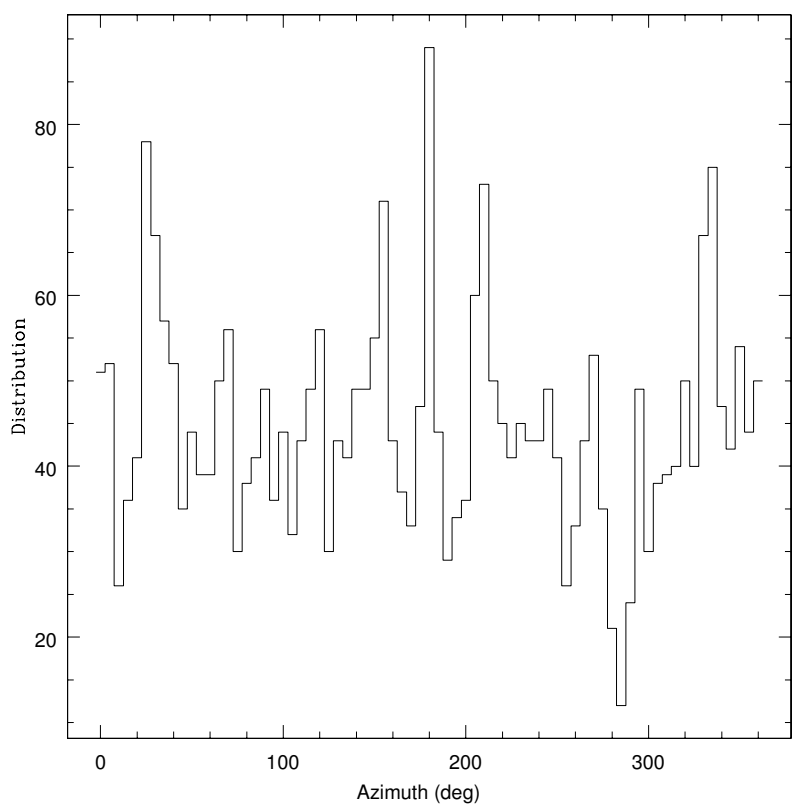

Fig. 5. Distribution of the orientations of the lines joining the $u$ and $z$ positions of all the CID-like observations.

individual images, at their highest resolution, are directly accessible through SkyServer ${ }^{2}$.

\section{CID over time}

Even when $2+$ observations secure the CID status of an object, that feature is far from being present in all its observations. One can therefore wonder how robust these detections are, even when confirmed at least once. So far, in this paper, only a tighter version of the criterion from the 2004 investigation has been used. Carrying out a consistency check among the successive CID-like observations would already be a substantial improvement.

2 http://skyserver.sdss.org/dr12/en/tools/chart/ 

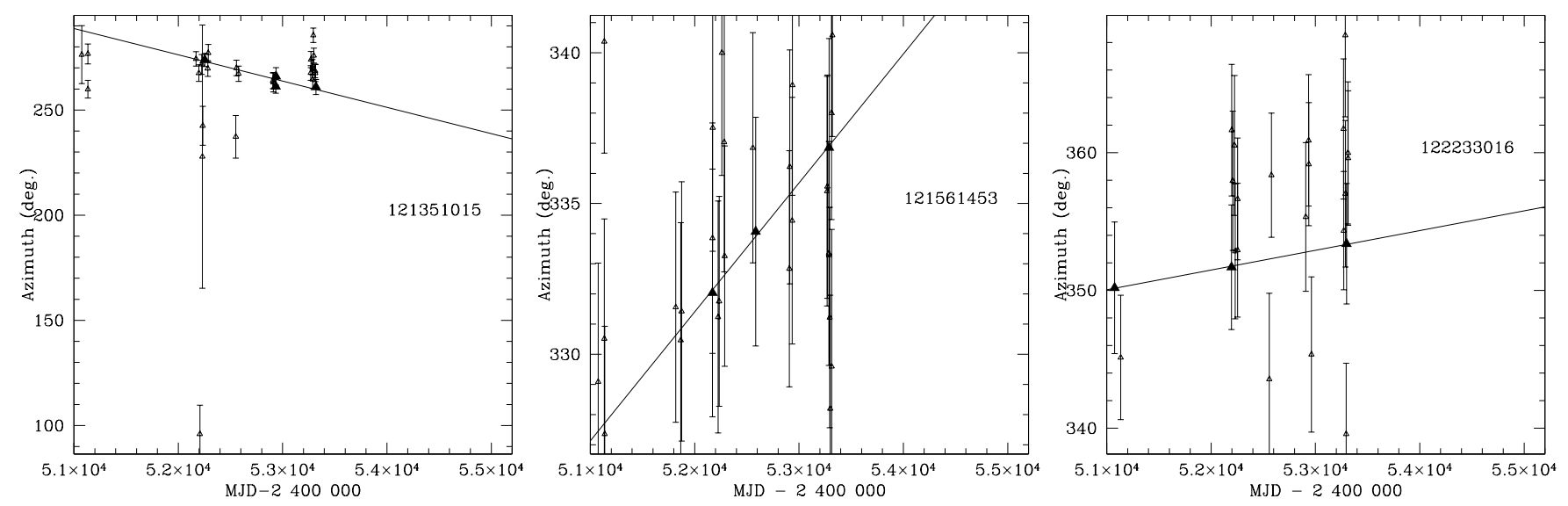

Fig. 6. Evolution of the azimuth over time. Open triangles denote observations without CID status. Filled triangles are valid CID observations and only these were included in the fit. The number is the unique thingId SDSS object number.

The line between the $u$ and $z$ positions essentially represent the line between the two stars. Unless a CID-candidate double star turns to be a short period binary (the observations cover at most 4178 days), the relative orientation based on the $u$ and $z$ positions should be fairly stable over time. For each of the 40 confirmed CID double stars, the orientations of all the validating observations are plotted in Fig. 4.

A vast majority of the CID candidates are unfortunately not confirmed by a second observation but one can nevertheless assess their global behaviour. For instance, the azimuth of all the CID candidates should be uniformly distributed over $0-360^{\circ}$. A $\chi^{2}$-test performed on the binned azimuths (Fig. 5) rejects the uniformity at the $99 \%$ confidence level. To assess whether this behaviour points towards any instrumental effect or not, a similar analysis was performed on the position angles of the 11057 members of the Double and Multiple Star/Component solutions of HIPPARCOS and Tycho Catalogues (ESA 1997). At the same confidence level, the uniformity hypothesis of the azimuths of the HIPPARCos resolved pairs is not rejected. So, why is it rejected with the SDSS CID? A Monte-Carlo simulation directly rules out any explanation based on the size of the sample.

Regardless of the coordinate and the filter, the four offsets of the CID observations are symmetrically distributed around 0 . However, whereas the distribution of the difference of offset in declination is bi-modal, the $\alpha *$ version, though similar, exhibits a third peak right on 0 . For all the objects in that third peak, the corresponding azimuth is either 0 or $180^{\circ}$ regardless of the offsets in declination, thus contributing to breaking the uniformity hypothesis. In terms of offset in declination, there is a tiny departure from symmetry right after 0 , thus causing a lack of azimuths around $280 \mathrm{deg}$. The objects identified in these bins do not share any common location in the sky.

Although Fig. 4 offers a good assessment of the CID nature of most of the 40 candidates, the successive observations do not provide any confirmation that any of these double stars is a binary. However, among the thirteen CID with $3+$ valid observations, there are three objects which also exhibit a monotonic evolution of the azimuth (Fig. 6). Actually, the evolution is not just monotonic: the azimuth of the valid CID observations follows a linear function of the time corresponding to $-4 . \pm 1.3$,

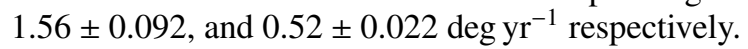

Inferring any orbital period from this azimuth gradient would be extremely speculative. SDSS J034237.97+010858.2 (object 12561453) was modelled as a DA white dwarf + M3 dwarf system (Rebassa-Mansergas et al. 2012a), $339 \pm 84$ pc away from us. Using their tentative masses and assuming an angular separation of $0.7^{\prime \prime}$ (which is a lower bound based on the $u$ and $z$ positions) yields a period of $3800 \pm 1500 \mathrm{yr}$ compared to the $230 \pm 15 \mathrm{yr}$ that we obtain assuming that our rate remains constant.

Could this evolution of the azimuth be a sign of a genuine binary? Generally speaking, no, unless the orbit is seen face on and the epochs are adequately distributed. However, for the three cases, the change of the azimuth does not exceed a couple of degrees over $1000+$ days. For any orbital segment short enough, this type of linear evolution of the azimuth would be very likely. Regardless of how appealing this explanation sounds, an alternative could be two stars passing next to each other in the plane perpendicular to the line of sight. Once again, this type of motion would not normally yield a linear change of the azimuth, except for the short path for which the linear approximation holds. As already stated in the title of this paper, CID are double stars, not necessarily binaries. One cannot exclude that they are made up of two stars accidentally on the same line of sight, although being far apart. However, if that is true for this technique, that is also true for the objects detected through their peculiar colours.

\section{Conclusions}

Whereas the possibility of detecting CID double stars with SDSS has been known for more than a decade, the absence of repeated observation (in particular of CID candidates) has prevented the validation of the technique, despite its easy setup. We have shown here that some CID are confirmed by at least a second observation. Furthermore, a consistency check confirms, with a criterion completely independent of the CID one, that the position angle of one component with respect to the other is stable over the time covered by the SDSS data (less than 4000 days). In three cases, a linear evolution of the azimuth of the stars is noticed over at least three distinct epochs. However, one cannot rule out the possibility that a high proper motion star is apparently passing by a distant star.

Acknowledgements. D.P. thanks Alain Jorissen for the stimulating discussions about the astrophysical aspects of these objects and A. Thakar from the SDSS helpdesk for his support in bypassing some early CasJobs limitations. We thank the referee, Andrei Tokovinin, for his valuable comments and suggestions. Funding for SDSS-III has been provided by the Alfred P. Sloan Foundation, the Participating Institutions, the National Science Foundation, and the US Department of Energy Office of Science. The SDSS-III web site is http://www. sdss3.org/. SDSS-III is managed by the Astrophysical Research Consortium for the Participating Institutions of the SDSS-III Collaboration including the University 
of Arizona, the Brazilian Participation Group, Brookhaven National Laboratory, Carnegie Mellon University, University of Florida, the French Participation Group, the German Participation Group, Harvard University, Instituto de Astrofísica de Canarias, the Michigan State/Notre Dame/JINA Participation Group, Johns Hopkins University, Lawrence Berkeley National Laboratory, Max-Planck-Institut für Astrophysik (MPA Garching), Max-Planck-Institut für Extraterrestrische Physik (MPE), New Mexico State University, New York University, Ohio State University, Pennsylvania State University, University of Portsmouth, Princeton University, the Spanish Participation Group, University of Tokyo, University of Utah, Vanderbilt University, University of Virginia, University of Washington, and Yale University. This research has made use of the Aladin sky atlas developed at CDS and of the SIMBAD database, operated at CDS, Strasbourg Observatory, France.

\section{References}

Abazajian, K., Adelman-McCarthy, J. K., Agüeros, M. A., et al. 2003, AJ, 126, 2081

Abazajian, K., Adelman-McCarthy, J. K., Agüeros, M. A., et al. 2004, AJ, 108, 502

Abazajian, K., Adelman-McCarthy, J. K., Agüeros, M. A., et al. 2005, AJ, 129, 1755

Abazajian, K. N., Adelman-McCarthy, J. K., Agüeros, M. A., et al. 2009, ApJS, 182,543

Adelman-McCarthy, J. K., Agüeros, M. A., Allam, S. S., et al. 2007, ApJS, 172, 634

Aihara, H., Allende Prieto, C., An, D., et al. 2011, ApJS, 193, 29

Alam, S., Albareti, F. D., Allende Prieto, C., et al. 2015, ApJS, 219, 12

Augusteijn, T., Greimel, R., van den Besselaar, E. J. M., Groot, P. J., \& Morales-Rueda, L. 2008, A\&A, 486, 843

Bolton, A. S., Schlegel, D. J., Aubourg, É., et al. 2012, AJ, 144, 144

Christy, J. W., Wellnitz, D. D., \& Currie, D. G. 1983, Lowell Observatory Bulletin, 167, 28

Clark, B. M., Blake, C. H., \& Knapp, G. R. 2012, ApJ, 744, 119

Doi, M., Tanaka, M., Fukugita, M., et al. 2010, AJ, 139, 1628

ESA 1997, The Hipparcos and Tycho Catalogues, ESA SP, 1200

Fukugita, M., Ichikawa, T., Gunn, J. E., et al. 1996, AJ, 111, 1748

Gunn, J. E., Carr, M., Rockosi, C., et al. 1998, AJ, 116, 3040

Heller, R., Homeier, D., Dreizler, S., \& Østensen, R. 2009, A\&A, 496, 191
Hogg, D. W., Finkbeiner, D. P., Schlegel, D. J., \& Gunn J. E. 2001, AJ, 122, 2129

Jayson, J. S. 2016, MNRAS, 457, 133

Kleinman, S. J., Kepler, S. O., Koester, D., et al. 2013, ApJS, 204, 5

Liu, C., Li, L., Zhang, F., et al. 2012, MNRAS, 424, 1841

Lupton, R. H., Ivezić, Ž., Gunn, J. E., et al. 2003, Proc. SPIE, 4836, 350

Morganson, E., Green, P. J., Anderson, S. F., et al. 2015, ApJ, 806, 244

Nebot Gómez-Morán, A., Gänsicke, B. T., Schreiber, M. R., et al. 2011, A\&A, 536, A43

Padmanabhan, N., Schlegel, D. J., Finkbeiner, D. P., et al. 2008, ApJ, 674, 1217

Pier, J. R., Munn, J. A., Hindsley, R. B., et al. 2003, ApJ, 125, 1559

Pourbaix, D. 2008, In A Giant Step: from Milli- to Micro-arcsecond Astrometry,

eds. W. J. Jin, I. Platais, M. A. C. Perryman (Cambridge University Press), 59

Pourbaix, D., Platais, I., Detournay, S., et al. 2003, A\&A, 399, 1167

Pourbaix, D., Ivezić, Ž., Knapp, G. R., Gunn, J. E., \& Lupton, R. H. 2004, A\&A, 423, 755

Pourbaix, D., Knapp, G. R., Szkody, P., et al. 2005, A\&A, 444, 643

Raymond, S. N., Szkody, P., Hawley, S. L., et al. 2003, AJ, 125, 2621

Rebassa-Mansergas, A., Gänsicke, B. T., Schreiber, M. R., Koester, D., \& Rodríguez-Gil, P. 2010, MNRAS, 402, 620

Rebassa-Mansergas, A., Nebot Gómez-Morán, A., Schreiber, M. R., et al. 2012a, MNRAS, 419, 806

Rebassa-Mansergas A., Zorotovic M., Schreiber M.R., et al. 2012b, MNRAS, 423, 320

Schlafly E. F., \& Finkbeiner D. P. 2011, ApJ, 737, 103

Schlegel, D. J., Finkbeiner, D. P., \& Davis, M. 1998, ApJ, 500, 525

Schreiber, M. R., Gänsicke, B. T., Rebassa-Mansergas, A., et al. 2010, A\&A, 513, L7

Silvestri, N. M., Lemagie, M. P., Hawley, S. L., et al. 2007, AJ, 134, 74

Smith, J. A., Tucker, D. L., Kent, S., et al. 2002, AJ, 123, 2121

Smolčić, V., Ivezić, Ž., Knapp, G. R., et al. 2004, ApJ, 615, L141

Sorokin, L. Y., \& Tokovinin, A. A. 1985, Sov. Astron. Lett., 11, 226

Stoughton, C., Lupton, R.H., Bernardi, M., et al. 2002, AJ, 123, 485

Szkody, P., Anderson, S. F., Agüeros, M., et al. 2002, AJ, 123, 430

Szkody, P., Anderson, S. F., Schmidt, G., et al. 2003a, ApJ, 583, 902

Szkody, P., Fraser, O., Silvestri, N., et al. 2003b, AJ, 126, 1499

Szkody, P., Henden, A., Fraser, O., et al. 2004, AJ, 128, 1882

Szkody, P., Henden, A., Fraser, O., et al. 2005, AJ, 129, 2386

West, A. A., Hawley, S. L., Bochanski, J. J., et al. 2008, AJ, 135, 785

West, A. A., Morgan, D. P., Bochanski, J. J., et al. 2011, AJ, 141, 97

York, D. G., Adelman, J., Anderson, J. E., et al. 2000, AJ, 120, 1579 